Chapter 11

\title{
Physical Properties of Seeds in Technological Processes
}

\author{
B. Dobrzański and A. Stępniewski \\ Additional information is available at the end of the chapter \\ http://dx.doi.org/10.5772/56874
}

\section{Introduction}

The physical properties of seeds and grain is a wide knowledge that can be useful in the farming, harvesting and storage or in processing such as drying, freezing and other. This knowledge is important in the designing of machinery to harvest and in preparation of processing chain from grain to food. Accurate design of machines and processes in the food chain from harvest to table requires an understanding of physical properties of row material. Considering either bulk or individual units of the agricultural material, it is important to have an accurate estimation of shape, size, volume, density, specific gravity, surface area, and other mechanical characteristics, which may be considered as designing parameters for food production. The measurement techniques allow computation of these parameters, which can than provide information about the effects of processing. Some of characteristics, such as colour, mechanical parameters, rheological properties, thermal and electrical resistance, water content and other physical quantities give excellent description of product quality.

\section{Physical properties of agricultural materials}

In a Newtonian sense, the physical properties of an object may include many varietals properties. Physical properties are determined for many raw materials and agricultural products, including seeds and grain, however, Barbosa-Cánovas et al. (2004) divided them into the following groups:

- Thermal properties such as specific heat, boiling point, conductivity, temperature, thermal transfer, diffusivity, and boiling point rise, freezing point depression.

- Optical properties, primarily colour, but also gloss and translucency. 
- Electrical properties, electric charge, electric field, electric potential, primarily conductivity, resistance, dielectric, conductivity, impedance, inductance, magnetic field, magnetic flux, and permittivity.

- Structural and geometrical properties such as mass, density, particle size, shape, volume, length, location, porosity, surface roughness, and cellularity.

- Mechanical properties (including strength, ductility, tension, compressibility, pressure, deformability moment, and textural properties, malleability,) and rheological properties (such as viscosity, flow rate, fluidity,).

- Others, including mass transfer related properties (diffusivity, permeability), surface tension, cloud stability, gelling ability, and radiation absorbance.

Seeds in general can display large compositional variations, inhomogeneities, and anisotropic structures. Composition can change due to seasonal variations and environmental conditions, or in the case of processed grain, properties can be affected by process conditions and material history.

Early physical property analyses of seeds and grain required constant uniform values and were often oversimplified and inaccurate. Nowadays, computational engineering techniques, such as the finite element method, are much more sophisticated and can be used to evaluate non-uniform properties (for example, thermal properties) that change with time, temperature, and location in grain that are heated or cooled. Improvements measuring the compositions of seeds are now allowing predictions of physical properties that are more accurate than previously.

\section{Physical methods for quality evaluation of seeds}

It was found that radiation in the near-infrared region of the spectrum can provide information related to many quality factors of agricultural products. Short wave radiations such as X-rays and gamma rays can penetrate through most seeds. The level of transmission of these rays depends mainly on the mass density and mass absorption coefficient of the material, that it allow find internal cracks invisible because of the seed coat (Dobrzański et al, 2003).

Nuclear magnetic resonance (NMR) is a technique that detects the concentration of hydrogen nuclei (protons) and is sensitive to variations in the concentration of liquid in the material. Although NMR imaging (MRI) has been used frequently in the medical field and other quality factors in grain has not been fully explored. Chen (1996) presents an overview of various quality evaluation techniques that are based on one of the following physical property: density, firmness, vibration characteristics, $\mathrm{X}$-ray and gamma ray transmission, optical reflectance and transmission, electrical properties, aromatic volatile emission, and nuclear magnetic response (NMR).

One of the most practical and successful techniques for nondestructive quality evaluation and sorting or separating impurities is the electro-optical technique, based on different optical 


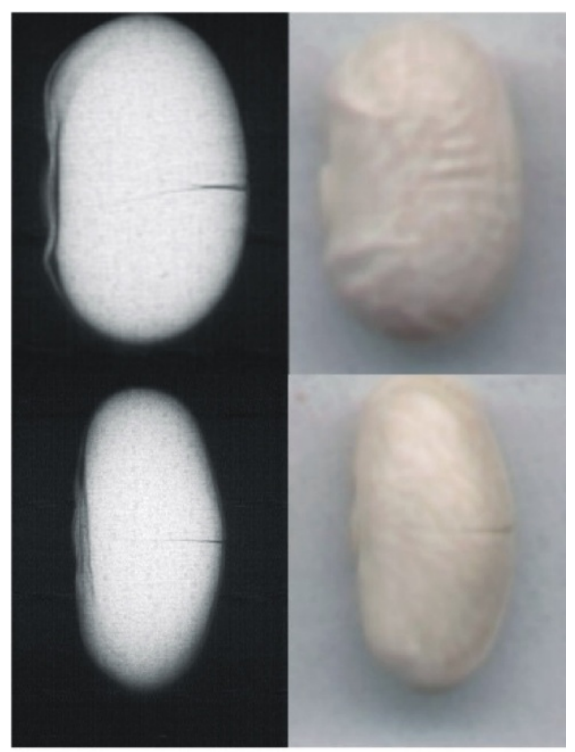

Figure 1. Invisible crack on the seed coat visible in X-ray image

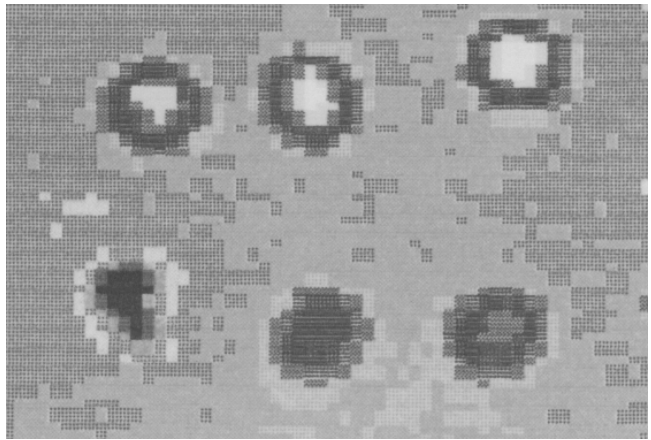

Figure 2. Thermal image of seeds after 3 hours of water soaking and imbibitions

properties of seed and impurities. Thus, determining such optical characteristics of seeds and grain can provide information related to quality factors of the product. Looking for more advanced method of germination capacity estimation, Dobrzański et al (2003) using thermal technique found slightly differences in temperature of dead and alive seeds, being soaked, that thermal properties should indicate live potential of seed in first hours of germination test. According to traditional germination test, that needs two weeks or more for forming germ (depends to species), thermography should be rapid technique for estimation of germination capacity. 


\section{Instrumental measurement of mechanical properties of seeds and grain}

There are many types of mechanical loading: puncture, compression, shearing, twisting, extrusion, crushing, tension, bending, vibration, and impact. And there are four basic values that can be obtained from mechanical properties tests: force (load), deformation (distance, displacement, penetration), slope (ratio of force to deformation), and area under the force/ deformation curve (energy). Mechanical characteristics included basic parameters (hardness, cohesiveness, viscosity, elasticity, and adhesiveness) and secondary parameters (brittleness or fracturability, chewiness, and gumminess).

The engineering terms based on these measurements are stress, strain, modulus, and energy, respectively. Stress is force per unit area, either of contact or cross-section, depending on the test. Strain is deformation as a percentage of initial height or length of the portion of sample subject to loading. Modulus of elasticity (tangent, secant, chord, or initial tangent) is a measure of stiffness based on the stress/strain ratio. Force and deformation values are more commonly used in food applications than stress and strain values, that are determined at seed loading.

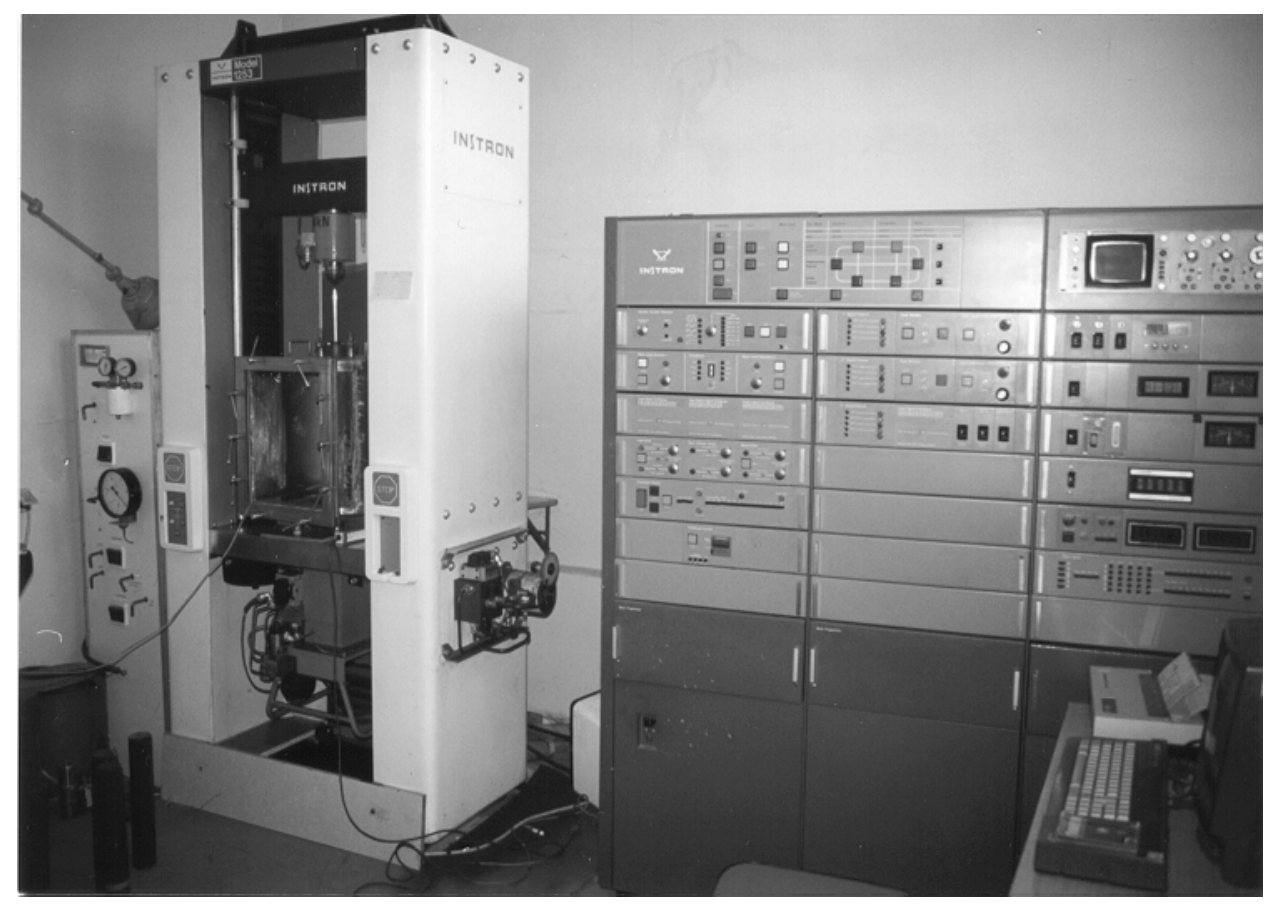

Figure 3. Universal testing machine Instron 1253 for fatigue test and dynamic load

Generally, determination of mechanical properties based on the high precision measurement, with expensive equipment (Fig. 3 and 4 ) requires a complete force-deformation curve. From 


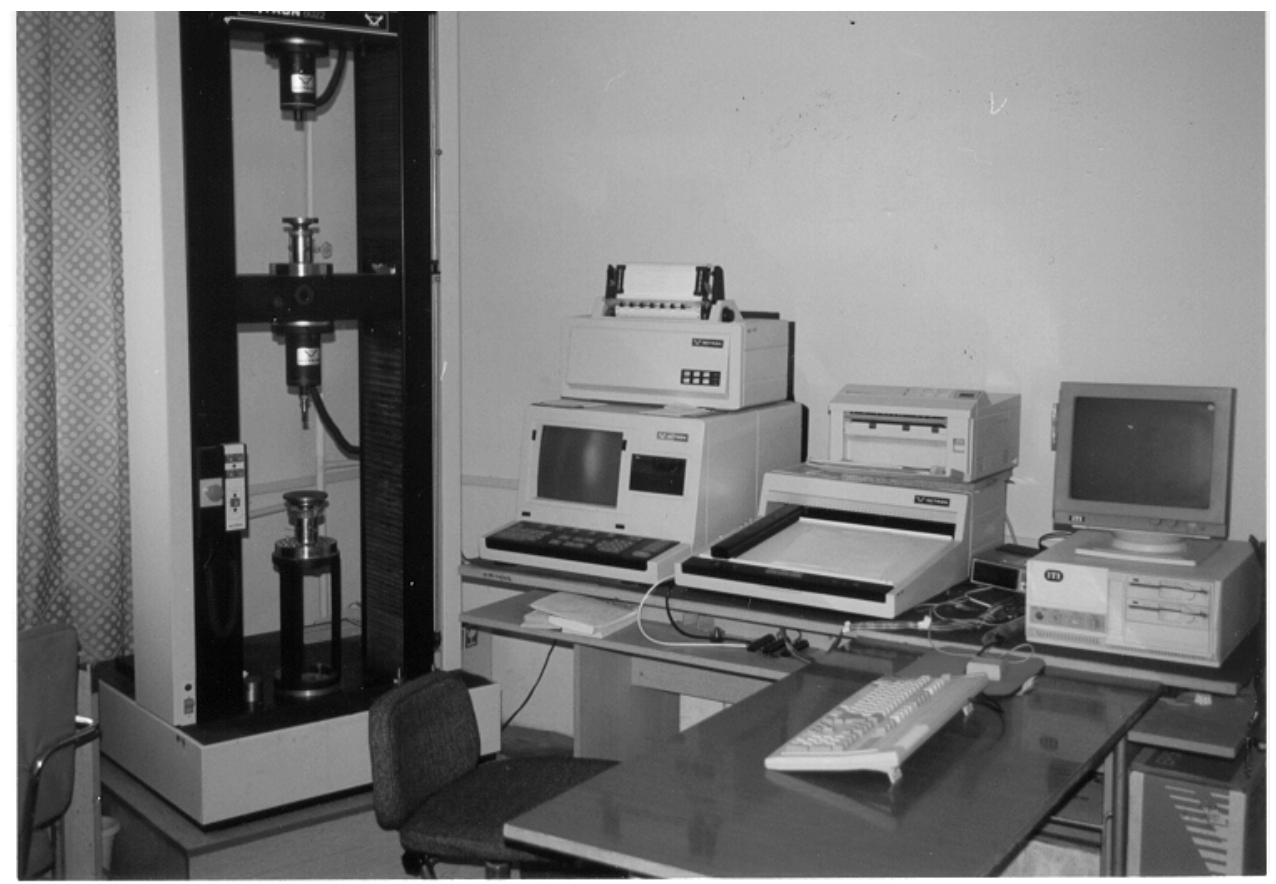

Figure 4. Universal testing machine Instron 6022 for quasi-static tests.

the force-deformation curve, stiffness; modulus of elasticity; modulus of deformability; toughness; force and deformation to point of inflection, to bioyield, and rupture, and maximum normal contact stress or stress index at low levels of deformation can be obtained. The force-deformation curve was frequently used in the study of physical properties of seeds and grain; however this measurement is not widely utilized in practice because of expensive and complicated procedures. A typical force/deformation characteristic for a cylindrical piece of tissue compressed at constant speed gives F/D curve for puncture tests look similar to compression curves. The portion of the initial slope up to point represents nondestructive elastic deformation; point is the inflection point where the curve begins to have a concavedownward shape and is called the elastic limit. The region before this point is where slope or elastic modulus should be measured. Beyond the elastic limit, permanent tissue damage begins. There may be a bioyield point where cells start to rupture or to move with respect to their neighbors, causing a noticeable decrease in slope (Abbott and Harker, 2003). (Dobrzański and Rybczyński, 2011 divided the measurements on direct (contact; compression, shear, impact, rebound) and indirect techniques (non-contact; vibration, sonic).

Seed hardness is related to the quality factors or behaviour of grain in relation to harvest, however, through use of simply test, only the maximum squeezing force has been frequently correlated with numerous quality factors. It is also well known, that including external and 


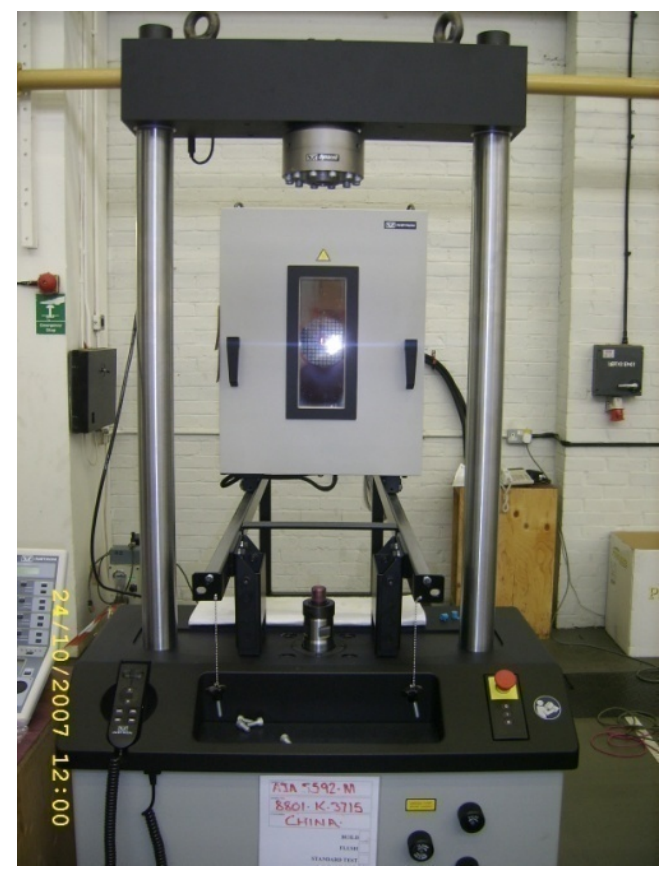

Figure 5. Temperature chamber mounted to 8872 Instron machine (High Wycombe)

internal properties of seed, the hardness should depends to the shape and size of seed; size and contact area with plate; rate of deformation; the way of seed fixing, and other procedures influenced a final accuracy. It is well known, that all mechanical properties and behaviour of agricultural materials are influenced by moisture and temperature, but universal testing machine with temperature chamber (Fig. 5) must be equipped.

\section{The cracking mechanisms of grain legume in technological processes}

The models describing some geometrical relations for different cracking mechanism were main aim of presented paper. In this study, the verify of cracking mechanism needs to determine the elasticity strain limit of cotyledon and the resistance of the seed coat to tension for various legume seeds. The change of shape as well as the mechanical values of the seed coat and cotyledon at various moisture contents, during swelling and drying were determined. This approach lead to an understanding of the possible causes and mechanisms that affect the cracking of the seed coat. Further, a major problem in the drying of legume seeds with heated air is the splitting of seed coat; affected by the temperature and moisture. The estimation of the effect of drying on the stress and strain development of the seed coat was determined at wide range of moisture. 
Legume seed quality is greatly affected by methods of harvesting and handling. In these operations, seeds are subjected repeatedly to many impacts on metal surfaces and against other seeds. Impacts may be result of threshing cylinder or rotor motion, the movement of buckets in vertical elevators, centrifugal discharging, the filling and discharging of screw conveyors, spouting and free-fall dropping (Palusen et al., $1981 \mathrm{a}, \mathrm{b}$ ). In nearly all handling operations, seeds are accelerated to some velocity and then discharged onto stationary objects or other grains.

Physical properties of agricultural products are needed for adequate design of processing machines but their specific applications should be clearly understood before determining it experimentally (Misra et al., 1981). The modulus of elasticity of seed is a physical property which has been suggested as an evaluation of firmness and hardness (Mac Donald, Jr.,1985). It is also an important property for determination of the stress cracks in seeds. However, in study of stress cracking due to drying of cereal grains from about 25 percent moisture content to about 14 percent moisture content, it is essential to understand the variation of modulus of elasticity according to wide range of moisture (Dobrzański, 1998; 2011).

The moisture content of seed coat and cotyledon influences the cracking mechanism and many researches have already published the results of mechanical properties including a modulus of elasticity for various seeds at a given moisture content and deformation rate. However, in order to clarify the mechanisms of seed cracking, it is necessary to carefully recognise the mechanical properties of cotyledon and seed coat separately.

The major problem in the drying of legume seeds with heated air is the splitting of the seed coat (Liu et al., 1989). Temperature and moisture gradients can cause stresses in the seed coat during drying. Overhults et al. (1973) observed the physical damage in soybeans during drying, in the form of indentations and cracks. This condition makes the beans susceptible to microbial attack during storage, and also reduces their germination potential (White et al., 1980). In view of these problems, there is need, specially in the legumineous seed industry, to find the optimum combination of drying parameters which will minimize seedcoat cracking (Mensah et al., 1984).

The following types of cracking mechanisms were observed during mechanical loading of the legume seeds:

- tension of seed coat caused by the shape deformation at axial compression,

- tension of seed coat caused by wet cotyledons sliding,

- shearing of dry seed coat caused by a force that smashed the seed in two cotyledons,

- crack opening displacement of the cotyledon under compression.

- splitting of the seed coat; affected by the high temperature and low moisture (a major problem in drying practice of grain legume). A rapid change of seed moisture during drying caused the shrinkage of seed coat leading to its stress. The splitting of the coat, caused by shrinkage, is frequently the most important mechanism of coat cracking. 


\section{Tension of seed coat caused by deformation of the seed compressed}

With the increase of moisture content seed become very plastic and even slight forces, which do not produce negative biological results, may cause considerable deformations of shape.

The description of the shape deformations of the legume seed compressed between parallel plates accomplishment needs some simplifying assumptions:

- the seed is a sphere surrounded by elastic shell,

- the compressibility of the cotyledon is very low that the volume is steady,

- the seed coat thickness is small that in geometrical calculations could be ignored,

- The seed compressed between parallel plates is circular in initial phase, deforming further in to the barrel of parabolic shape at large deformation.

Using the assumption of constant volume, make it possible to determine the changes of surface area describing the spherical body being compressed between parallel plates. When the deformation is slight the sphere does not lose its shape and hence it was assumed that lateral surface of barrel created by compressing the opposite cups of sphere will also be spherical in its shape.

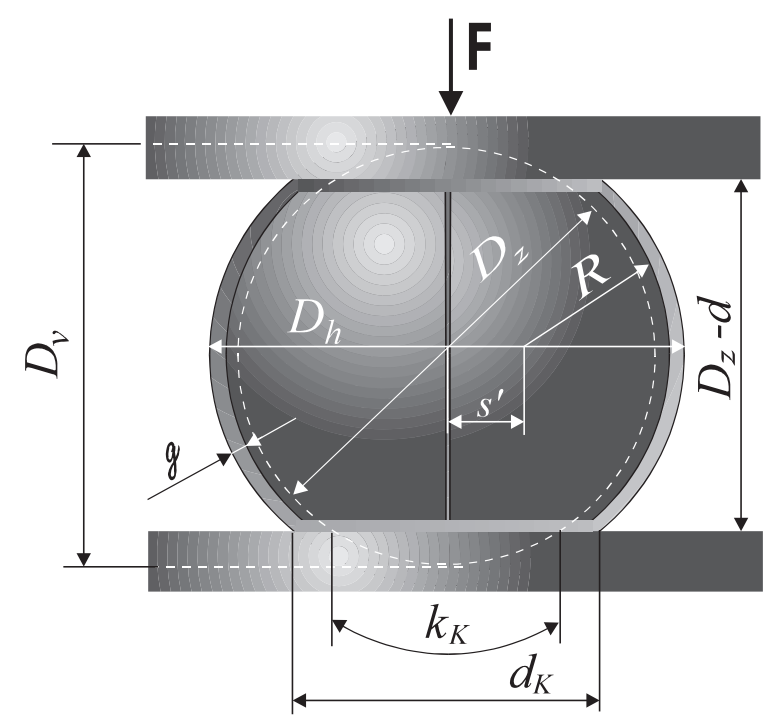

Figure 6. The shape deformation of seed at axial compression.

In initial phase of a shape deformation of viscoelastic seed was observed. Further cotyledons deformation causes seed coat filling, and when this process completed, an increase of the inner pressure leads to the seed coat tension; till its disruption. Assuming that during seed coat 
tensions the pressure necessary for shape deformation is constant, the increase of pressure $\Delta p$ ${ }_{w}$ resulting in the seed coat cracking can be determined from the force-deformation curve.

Substituting characteristic values, taken form the force-deformation curve obtained in compression tests of legume seeds and the diameter of contact area as well as diameter of barrel calculated using geometrical formulas presented in previous papers (Dobrzański, 1998) it is possible to determine the modulus of elasticity of seed coat $E_{\text {om }}$ from the following equation:

$$
E_{o m}=\frac{D_{h} \Delta p_{w}}{\Delta D_{h}}\left(\frac{\left(0.5 D_{h}+g\right)^{2}+\left(0.5 D_{h}\right)^{2}}{\left(0.5 D_{h}+g\right)^{2}-\left(0.5 D_{h}\right)^{2}}+v\right)
$$

The above method enabled the interpretation of the force-deformation curve for large deformation of non-elastic wet seeds where mechanical strength is mainly connected with the resistance of the seed coat to tension.

\section{Tension of wet seed coat caused by the sliding displacement of the cotyledons}

The compression of seed leads the shape deformation, however, low increase of lateral surface at seed strain under $\varepsilon<0.3$ proves, that there are any stresses of it. On the other hand, sliding of wet cotyledons during compression caused larger shape deformation of seed leads to tension of the seed coat and stress.

Sliding cotyledons surrounded by the seed coat caused tension strain of the seed coat $\varepsilon$ or which can be calculated from simple equation based on geometrical relations according to the figure 7:

$$
\varepsilon_{o}=\frac{\pi r_{w}-k_{U}+d_{U}}{\pi r_{w}}
$$

Substituting geometrical relations for above values of formula (2) it is possible to determined strain of the seed coat largest diameter in cross section of body formed by sliding of cotyledons:

$$
\varepsilon_{0}=1-\frac{1}{180} \arccos \frac{D_{z}-2 d}{D_{z}}+2 \frac{\sqrt{\left(\frac{D_{z}}{2}+\frac{D_{z} d}{D_{z}}\right)^{2}-\left(\frac{D_{z}}{2}\right)^{2}}}{\pi D_{z}}
$$

The change of seed shape during compression caused increase of the surface area surrounding cotyledon. A sphere surrounded by a shell imitating a seed is deformed on both sides and 
change the shape into a barrel when compressed between parallel plates. When the deformations are slight the sphere does not lose its shape and hence it was assumed that the lateral surface of the barrel created by compressing opposite cups of sphere will also be spherical.

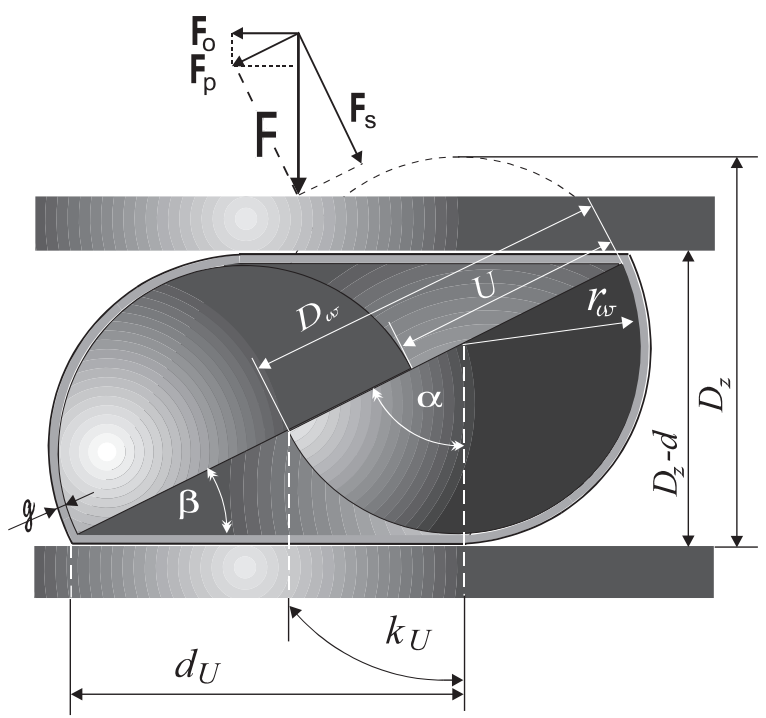

Figure 7. Tension of seed coat caused by the sliding displacement of the cotyledons.

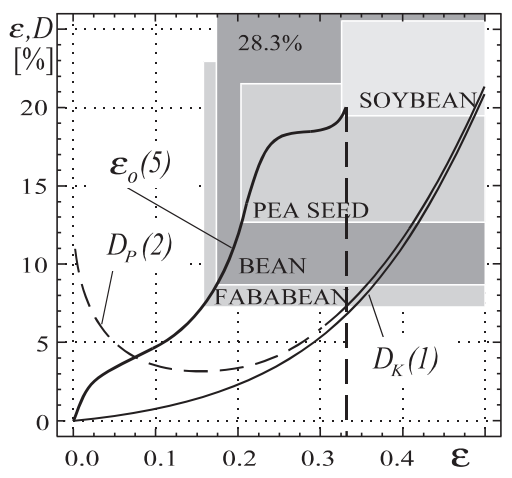

Figure 8. Increase of the seed diameter caused by the shape deformation $D_{K}, D_{p}$ and cotyledons sliding $\varepsilon_{0}$.

Circumferential strain $\varepsilon_{o}$ at tension caused the sliding displacement of the cotyledons determined using formula (3) is represented on the figure 8. The increase of shape deformation is represented by barrel diameter of parabolic $D_{P}$ and circular shape $D_{k}$. Both diameters increases with an increase of axial deformation, however, almost $10 \%$ of the increase was 
obtained for 0.4 axial strain. In comparison, cotyledon sliding caused coat strain $\varepsilon_{o}$ up to $15 \%$ for axial strain $\varepsilon$ close to 0.2 only. It suggests that sliding may involve rupture easier than shape deformation at compression. The range of legume seed coat elongation at rupture for various moisture are presented on Figure 8. It is easy to conclude, that fababean and bean seed coat is most influential on tension at axial compression and sliding of the cotyledons. At 0.18 of axial strain caused cracking of the seed coat of some fababean and bean.

Fababean (Vicia faba L.), soybean (Glicine hispida Max.), bean (Phasoleus L.), and pea seed (Pisum sativum L.) cultivars were studied to mechanical resistance of seeds subjected to load at different rates of deformation. The resistance of legume seeds to compression was determined over a wide range of moisture contents. The diameter of seed and shape deformation under compression, as an additionally measurement, were determined with strain gauge. Most resistant species of legume seeds is soybean, for which the seeds could not be damaged in this way. The determination of the cracking mechanism of the cotyledon was carried out at low moisture only with single cotyledons -- a half of bean was compressed between parallel plates at various rates of cross-head movement.

The resistance of seed coat to tension was studied for all seeds at wide range of moisture. The drying test was conducted to evaluate the effect of drying parameters on the stress and strain development of the seed coat. To evaluate the effect of drying conditions on the splitting of seed coat, the samples were dried with air. The time-strain curves at different drying conditions were obtained with 2630-107 Instron strain gauge. Three air velocities: $4.3 ; 10.5 ; 15.6 \mathrm{~m} / \mathrm{s}$ and four temperatures: $22 ; 42 ; 52 ; 62^{\circ} \mathrm{C}$ were used as parameters of the drying tests.

The results obtained from compression tests of cotyledons of fababean cultivars as an example was presented in previous paper (Dobrzański, 1998). Different values of cracking force for each cultivar indicate that the strength of cotyledons differ. However, the modulus of elasticity is almost the same and there is no significance difference among $(\mathrm{p}=0.05)$ the cultivars. This was also noted for other legume species.

\section{Shearing of the seedcoat at threshing of dry seed}

The shearing of dry seedcoat most frequently observed mechanism of bean cracking at trashing, is caused in dry seed only, by a force which is parallel to the plane of cotyledons contact. This mechanism is characteristic also for rape seed and other legume seeds such as pea seed, french bean, soybean and fababean, which covers under the seed coat two cotyledons. Some of dry seed during processing are separated two parts. It may be result of threshing cylinder or rotor motion, centrifugal discharging, the filling and discharging of screw conveyors, sprouting and free-fall dropping. Each part consists the cotyledon and half of the seed coat, which is shearing by a force parallel to the plane of contact of the cotyledons (Fig. 9).

Approximately cross section area of the seed coat, for oblong seeds such as: soybean, bean and french bean, was determined according to the figure 4 from the formula: 


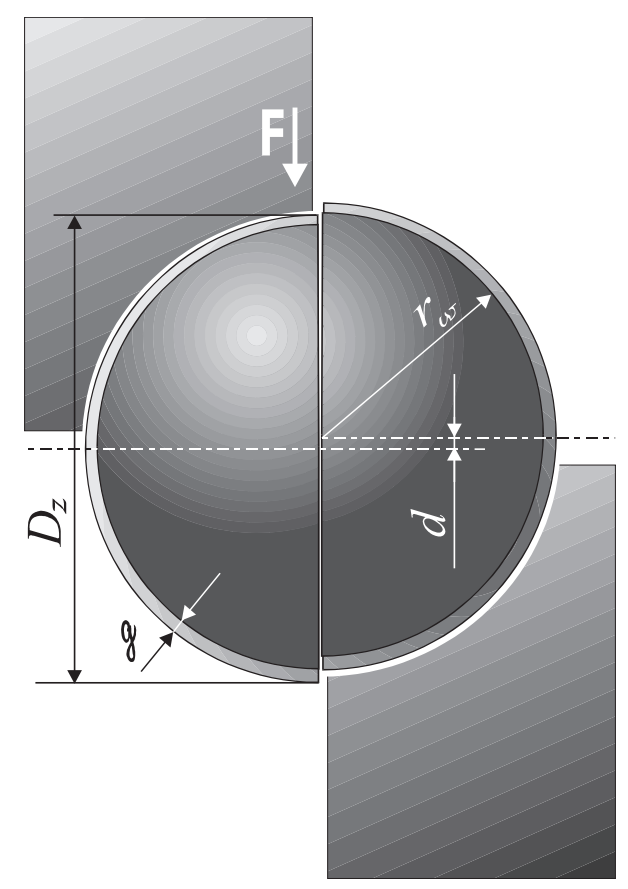

Figure 9. Shearing of the seed coat.

In this mechanism of dry seed cracking the force $F$ made work $L$ at deformation $d$, leads to the seed coat shearing, which for elastic body may by calculate using simple formula:

$$
L=\frac{F d}{2}
$$

Both of these values are determined in experimental test presented on Figure 9. According to the energy conservation law it is possible to determined permissible velocity limit $V_{p}$, for which the seed of known mass $m$ did not cause the seed coat cracking. Equating both energies: kinetic energy of spouting seed and work of cracking at seed coat shearing following formula was obtained:

$$
V_{p}=\sqrt{\frac{F d}{m}}
$$

This method of velocity limit $V_{p}$ calculation covers some simplifying assumptions, however, it allows to estimate the seed cracking of dynamic processes frequently meets in practice, by simply way at quasi-static test. 


\section{Shrinkage stress of the seed coat at drying}

The effect of moisture content on expansion volume of soybean was shown in the experimental results presented in previous paper (Dobrzański, 1996). Seed dimensions increased during swelling for all cultivar. More distinctly effects of moisture content on dimensions of soybean were observed in the initial phase of swelling. Further seed's filling, leads to the seed coat tensioning. Similar behavior and significant decrease of volume in other grain legume was observed (Fig. 10).

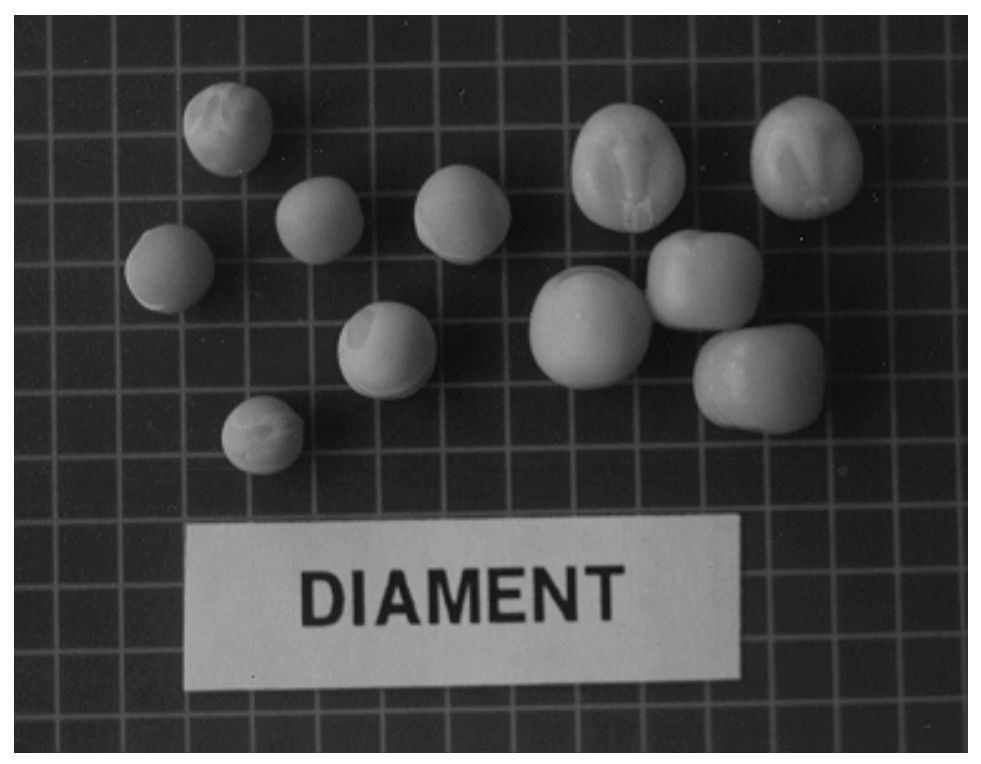

Figure 10. Volume expansion of pea seed (v. Diament) caused by water imbibitions during soaking.

The water vaporizing from the seed is limited by coat and the results presented in previous paper (Dobrzański, 1996) proved that this process was not rapid. On the other hand, all dimensions of seed decreased at this process and exponential relationship was observed.

Rapid change of moisture content of seed coat during drying caused the shrinkage of coat leading its stress. Highest shrinkage for rudest condition of drying was observed. When the drying conditions were milder, the shrinkage of the seed coat was lower. The Figure 11 presents a model of spherical seed in the stream of air $T_{t V}$. The wet seed of volume $V_{K w}$ is surrounded by the seed coat of diameter $D_{W}$. During drying the seed coat try to reduce its volume to $V_{K s}$ following its surface and diameter $D_{s}$.

The shrinkage of the seed coat on wet seed of the large volume leads the stress, which involve increase of the inner pressure $p_{w}$ of incompressibility wet cotyledons. 
In order to determine strength criteria for tension seed coat at drying, Dobrzański (1998) found that, it is sufficient to estimate circumferential stress $\sigma_{o}$ in cross section of seed. He determined the circumferential stress in the seed coat using Lamé equation concerning radial strain and stress of a pipe subjected to the inner pressure $p_{w}$ :

$$
\sigma_{o}=\frac{p_{w}\left(0.5 D_{W}^{2}+D_{W} g+g^{2}\right)}{D_{W} g+g^{2}}
$$

During drying, the circumferential shrinkage stress $\sigma_{o}$ must meet the strength criteria:

$$
\sigma_{o} \leq k_{r}
$$

Taking into account, that stress of the seed coat $\sigma_{s}$ at drying depends to shrinkage strain $\varepsilon_{s}$ and modulus of elasticity of the seed coat $E_{o}$ it must be verify experimentally.

The single seed presented on figure 11 is in the stream of hot air, however, most seeds at real condition are in the layer being in contact to other. It limits inflow of air to some parts of seed in contact to other (Fig. 11).

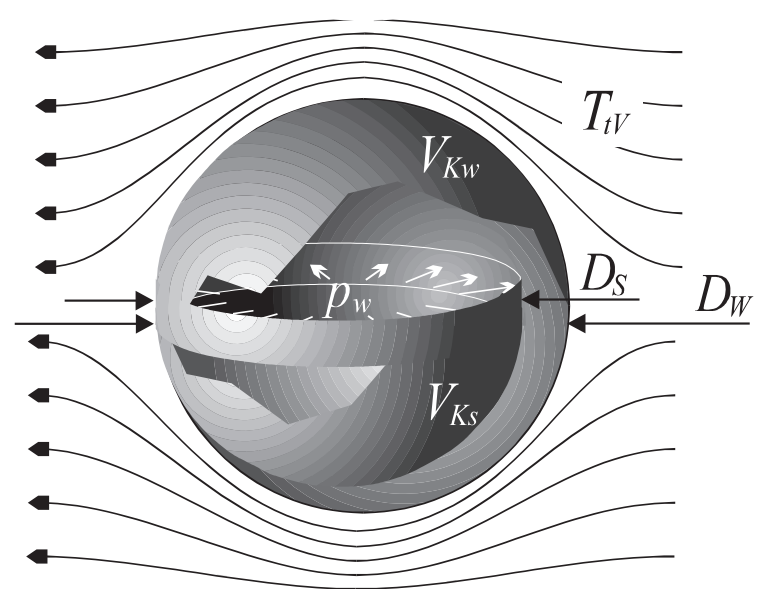

Figure 11. The shrinkage of the seed coat in the stream of air.

Hot air caused the shrinkage of part available for the stream of air. Residual part of seed coat being in the contact to other seed keeps higher moisture content. Dobrzański and Szot, 1997; Dobrzański, 2011) found that mechanical strength of seed coat decreases with the increase of moisture content. Finally, shrinkage of dry seed coat caused tension of wet part of it. Figure 12 presents the cross section of two seed being in the contact. 


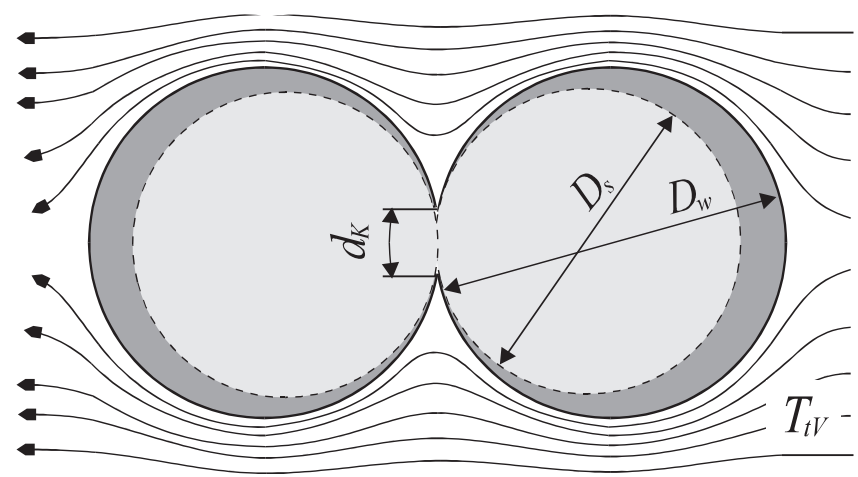

Figure 12. The shrinkage of part of seed coat in the stream of air.

The circle diameter $D_{W}$ decrease to $D_{S}$, however only the part $\left(D_{W}-d_{K}\right)$ of the seed coat is being subjected to the shrinkage. It allows determination of the shrinkage strain $\varepsilon_{s}$ of dried part of the seed coat:

$$
\varepsilon_{S}=\frac{\pi\left(D_{W}-D_{S}\right)}{\pi D_{W}-d_{K}}
$$

The shrinkage leads to the stress $\sigma_{s}$, which depend to the modulus of elasticity of dry seed coat $E_{o s}$ :

$$
\sigma_{s}=E_{o S} \varepsilon_{s}
$$

The shrinkage of dry seed coat caused tension $\sigma_{o}$ of wet part $d_{K}$. Determining strain $\varepsilon_{o}$ of wet part of the seed coat subjected to extension and its modulus of elasticity $E_{o W}$, it is possible to estimate the tension strength limit $\sigma_{u}$ :

$$
\sigma_{u}=E_{o W} \varepsilon_{o}
$$

For safe condition of drying must be fulfil an inequality: •

$$
\sigma_{u}>\sigma_{s}
$$

For this purpose, the shrinkage stress $\sigma_{s}$ and strength $R_{m}$ (Fig. 14) strain $\varepsilon_{s}$, ultimate elongation at rupture $\varepsilon_{o}$ and the modulus of elasticity $E_{o}$ (Fig. 13) must be determine experimentally in wide range of moisture. 


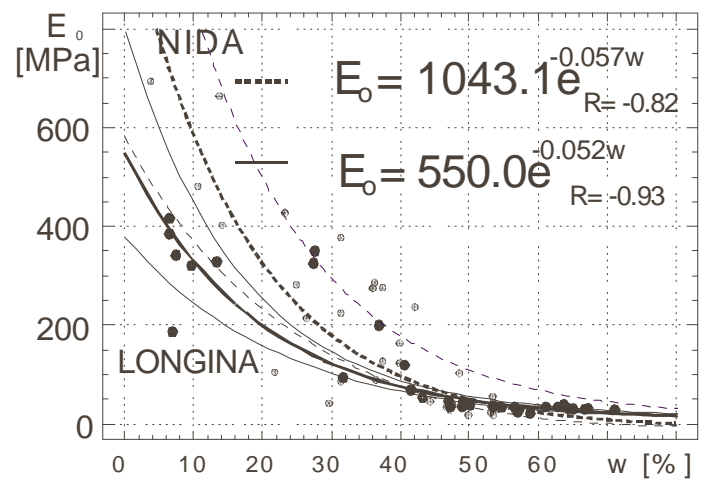

Figure 13. Modulus of elasticity of the seedcoat for Nida and Longina varieties in the range of moisture from 6 to $70 \%$.

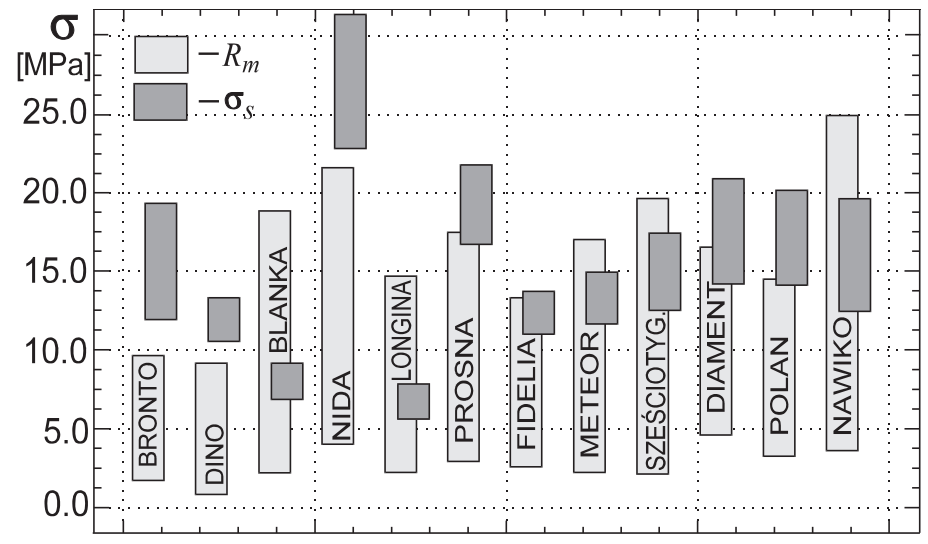

Figure 14. The range of seed coat strength $R_{m}$ to tension and shrinkage coat stress $\sigma_{s}$ at drying for fababean (Bronto, Dino) bean (Blanka, Nida, Igolomska, Prosna), pea seed (Fidelia, Szesciotyg, Diament, Meteor) and soybean (Polan, Nawiko).

A preliminary study was conducted to establish the time required for strain of seed coat after being dried to final moisture content. The second series of drying tests was conducted to evaluate the effect of drying parameters on the stress and strain development of seed coat. To evaluate the effect of drying conditions on the splitting of seed coat, the samples were dried with air. The seed coat ring samples were prepared according to the method elaborated by Dobrzański (1998).

The results obtained at tension of the seed coat shoved that, the increase of moisture content involves the decrease of force at breakage. Cultivar's differentiation of seed coat strength in tension test was observed. The results obtained in all experiments concerning with strength of seedcoat proved that frequently the stress was higher than strength of seed coat (Fig. 14). 
Probably, it is one of the most important reason of the seedcoat cracking during drying. The rapid decrease of air temperature during drying causing additional shrinkage of seed coat, clarifies the origin of the coat cracking after disconnection of the heating set in dryers, observed in practice. The highest shrinkage for all cultivar at most severe drying condition was observed. The value of force induced by the shrinkage depended also on drying conditions. Drying of the seed coat at temperature of $62^{\circ} \mathrm{C}$ gave highest shrinkage stress. Further drying with the cold air caused the increase of seed coat shrinkage for $25 \%$ more. This phenomenon was observed for other cultivars. Increase of moisture content leads to a decrease of force at breakage. The shrinkage of the seed coat during drying was more than $26 \%$ for all seeds.

The theoretical considerations of cracking mechanisms allows to verify some models describing geometrical relations during shape deformation and drying. The modulus of elasticity of the seed coat was determined at compression and shape deformation. However, this method enabled the interpretation of the force-deformation curve for seed covering moisture over $12 \%$. On the other hand, the seed strain (under 30\%) leads shape deformation without stress in the coat. For large deformation of wet seeds mechanical strength is mainly connected with the resistance of seed coat to tension. Sliding of the cotyledons is one of most important reason of seed coat cracking for seed over $20 \%$ of moisture content.

Shrinkage of the seed coat at drying leads to its stress and splitting. The results obtained in all experiments concerning with strength of seed coat proved that frequently the stress $\sigma_{s}$ was higher than strength $R_{m}$ of seed coat. Probably, it is one of the most important reason of the seed coat cracking during drying. The additional shrinkage of the seed coat clarifies the origin of cracking, after disconnection of the heating set, frequently observed in practice.

\section{Postharvest rapeseed quality according to seeds physical properties}

Physics of plant material concentrates on estimation of physical features important for material quality during vegetation, harvest, transport, storage and processing. It also studies microstructure of plant materials and it influence on differentiation of properties. Physics of plant materials develops methods of estimation of damage in agricultural products and the mechanisms of its appearance taking into account external factors. The variations between the varieties are also under investigation. All the data collected from the above mentioned measurements allows elaborating technological improvements for preventing the unfavorable effects of damage. This in consequence increases the quality of raw materials for food production.

Agrophysical metrology allows widen and deeper analysis of processes undergo during each stage of agricultural production with a special emphasis on harvesting and postharvest operations, deals with adaptation, improvement and designing measuring methods, which can be used to explain quantitative and qualitative changes, which take place in agriculture. It develops methods as well as equipment for measuring, monitoring and analyzing the time and space variability of physical parameters of agricultural materials subjected to agrotechnical and technological processing. 
The investigations were performed in field conditions and some simulations were also a subject of investigations in laboratory. Samples were collected at regular storage rooms and driers. Physical parameters of rapeseed were obtained in compression tests of single seeds. Compression curves were registered and analyzed with numeric methods to calculate apparent modulus of elasticity and strength of seeds.

Monitoring of quality of storied seeds was made in specially constructed storage silos which allowed measurement of pressure and temperature. The influence of seed moisture content on changes of quality within the storage time was described.

The investigations showed considerably differentiated quality features (percentage of broken seeds) of rapeseed within the whole typical process of their postharvest processing. The amount of broken seeds increased even two times comparing samples taken from harvester (threshed seeds) and samples taken from storage room of dry seeds.

\begin{tabular}{ccc}
\hline OPERATIONS & $\begin{array}{c}\text { BROKEN SEEDS } \\
\text { Min [\%] }\end{array}$ & $\begin{array}{c}\text { BROKEN SEEDS } \\
\text { Max [\%] }\end{array}$ \\
\hline Unloading of threshed seeds & 1,6 & 7,5 \\
\hline Storage room of wet seeds & 1,6 & 8,0 \\
\hline Before drying & 1,5 & 8,9 \\
\hline After drying & 1,7 & 9,1 \\
\hline Storage room of dry seed & 3,2 & 8,2 \\
\hline
\end{tabular}

Table 1. The changes of the amount of broken seeds during rapeseed postharvest processing

Investigations showed considerable decrease of quality features of rapeseed during harvesting and postharvest operations. The amount of broken seeds considerably depended on their moisture content. The dependence between moisture content of seeds and their resistance to external loads resulted in quality losses was proofed. The most resistant seeds were observed at $6 \%$ of moisture content and decreased both with increased and decreased of seed moisture.

Quality features of stored seeds depended on their moisture content, physical state of seed cover (broken or untouched), pressure and temperature inside silo. Safe time of storage was longer for dryer seeds. In that case of unbroken seeds biological activity was lower as well as biochemical processes underwent slower. The methods could be used to evaluate physical condition of rapeseed. All the methods allowed to monitor changes of quality features of rapeseed as raw material for oil production.

According to the results obtained it can be stated that the most negative influence on quality features of rapeseed (amount of broken seeds) had harvest operation. The amount of broken seeds increase during successive postharvest operations i.e.: transport, cleaning, drying, however the initial quality parameters had considerable significance for final quality of raw material for oil production. The methods used allowed a precise monitoring of changes of rapeseed quality parameters. 


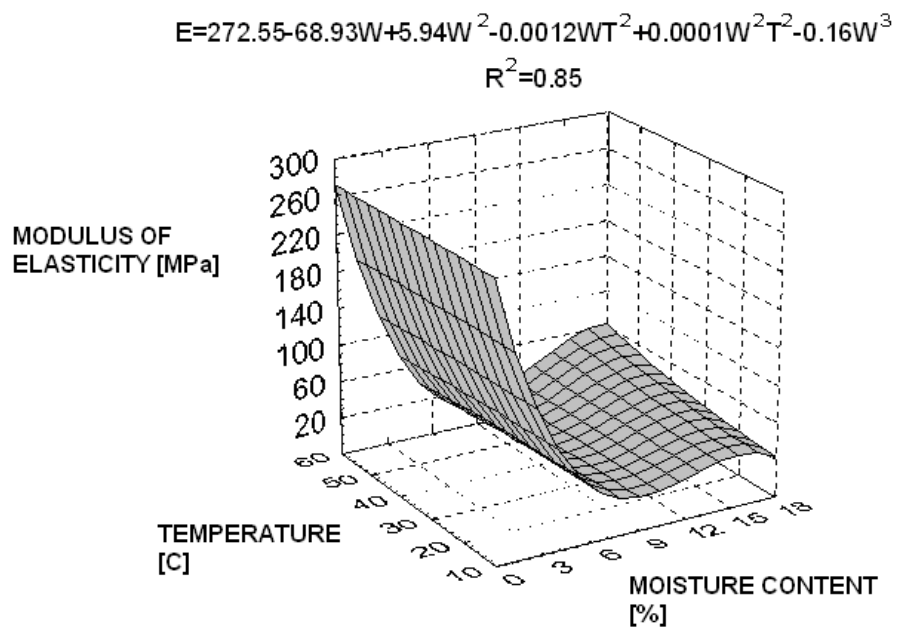

Figure 15. Influence of moisture content and temperature on rapeseed apparent modulus of elasticity

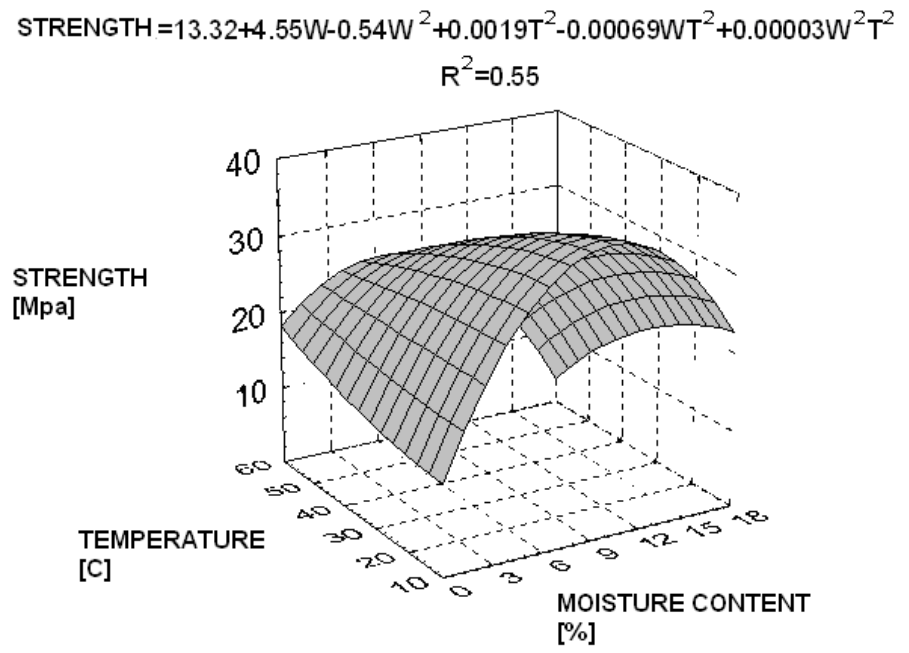

Figure 16. Influence of moisture content and temperature on rapeseed strength

Laboratory studies of mechanical strength of rapeseed allowed indicating range of "safety" moisture content for handling. Seed of $6 \%$ of moisture content had the highest strength. Both the increase and the decrease of moisture content negatively influence strength features of seed. The influence of temperature was much lower than the influence of moisture content in the case of modulus of elasticity as well as in the case of strength. Some methods as well as results could be utilized also for other oilseeds i.e. soybean. 


\section{Technological parameters of lentil seeds}

Lentil is not a significant plant from the point of view of the economy and agronomy (crop rotation), however have unique nutritional properties and most of all is regarded as so called healthy food. Therefore lentil sees become more and more popular and are sought after and desired by consumers as an additive or raw material for the food, pharmaceutical and chemical industry. Physical parameters of such "exotic" plant are under investigation as they are important from the technological point of view.

Air-dry lentils has a mass of 1000 seeds $49.36 \mathrm{~g}$ for the variety Tina and $48.05 \mathrm{~g}$ for the variety Anita. This parameter increases with increase of seeds moisture content and at $24 \%$ seeds achieve mass of $57 \mathrm{~g}$ for Tina and $58 \mathrm{~g}$ for Anita respectively. The high correlation coefficient was noticed in case of both varieties i.e. 0.987 for Anita and 0,975 for Tina.

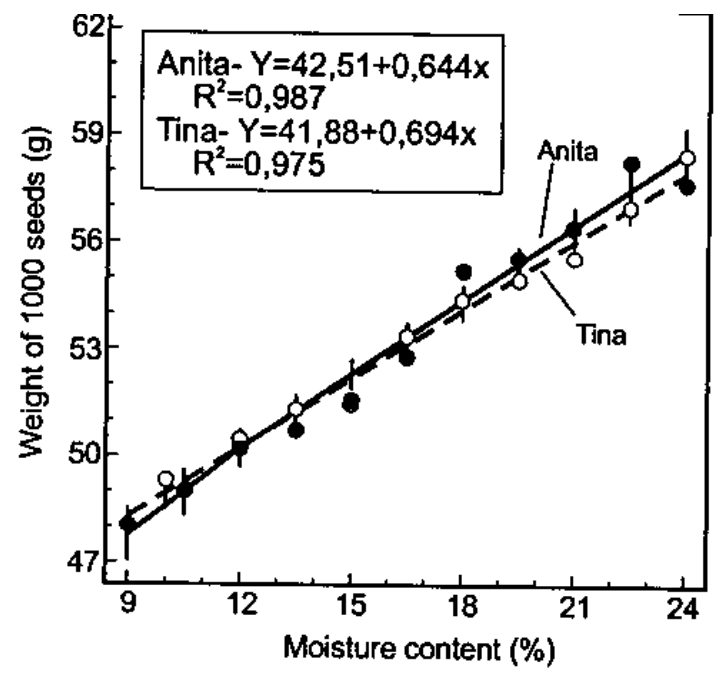

Figure 17. The influence of moisture content of seeds on their weight

Similarly, with an increase of moisture content of the lentils seed its porosity increase. This characteristic was observed for both tested varieties and porosity parameter ranged from 44 to $47 \%$ within moisture content range of $8-24 \%$. The high correlation coefficient was noticed in case of both varieties i.e. 0.931 for Anita and 0,927 for Tina. The differences between varieties were statistically significant and higher values of this parameter was observed for Tina variety.

Density of lentil seed decreased with an increase in their moisture content. The maximum value of this parameter was observed at $8 \%$ moisture content for the variety Tina was $806 \mathrm{~kg} /$ $\mathrm{m}^{3}$. The lowest recorded value is $747 \mathrm{~kg} / \mathrm{m}^{3}$ and this value was similar for both varieties at the humidity of $24 \%$. Also in this case the high correlation coefficient was noticed for both varieties i.e. 0.959 for Anita and 0,913 for Tina. 


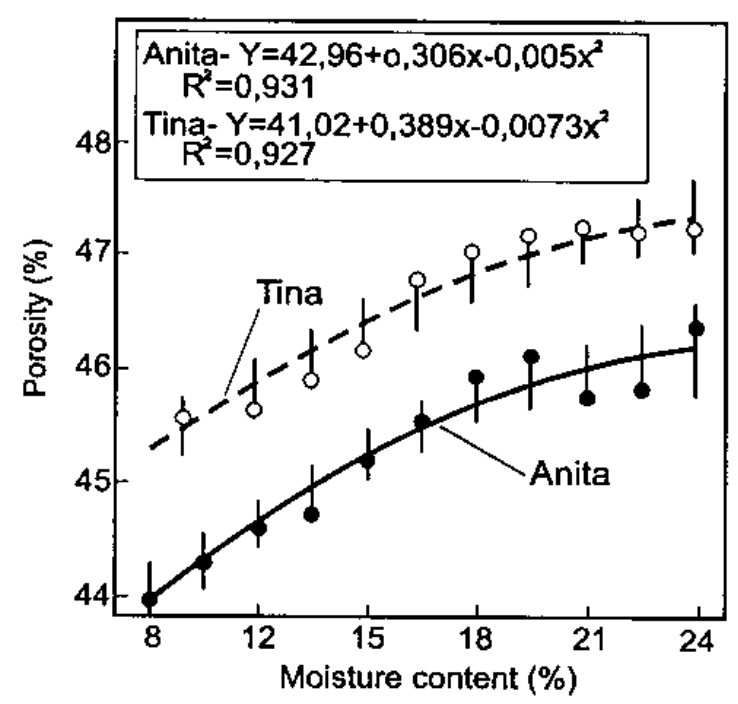

Figure 18. The influence of moisture content of seeds on their porosity

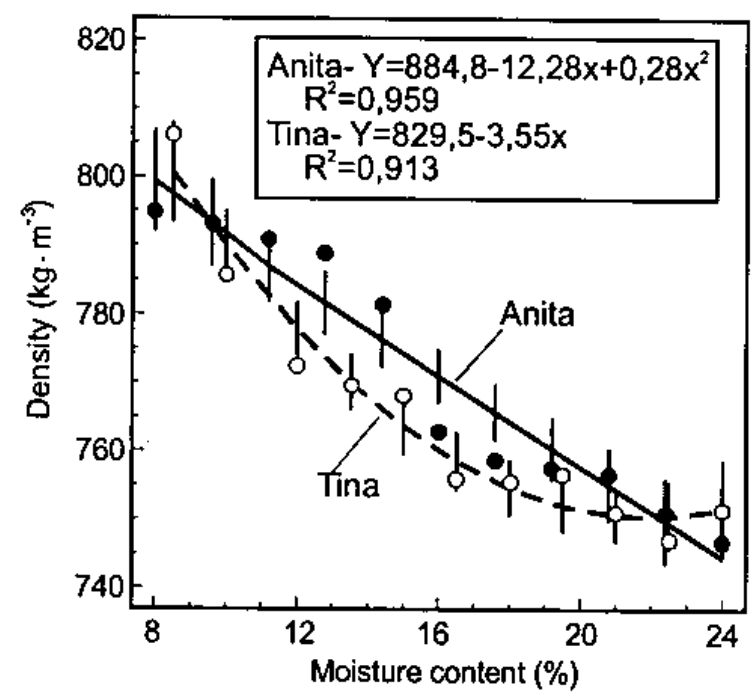

Figure 19. The influence of moisture content of seeds on their density

With the increase of moisture content both angle of repose, and slippery increase. These values of the angle of repose was measured in the range of $27.5^{\circ}$ for air-dry seeds of Tina variety to $34 \stackrel{\circ}{ }$ for the same variety at moisture content of $24 \%$. 


\section{Sweet corn kernels mechanical properties and the effect of harvest time, storage and blanching}

Sweet corn attains its consumption and processing ripeness within 24 to 28 days from blooming that is from the appearance of browning that manifests ripeness. In the phase of late milk ripeness, kernels assume yellow-gold colouring. The time of harvest not only determines the physical properties of sweet corn cobs and kernels, but also generally affects the mechanical strength of the kernels. Although the time of harvest mainly affects the mechanical properties of kernels, one should also expect chemical changes related to the ripeness stage. The differences between the mean values of the cutting force were statistically significant and varied within the range from 34.2 for harvest term I to $10.3 \mathrm{~N}$ for term III (Fig. 20).

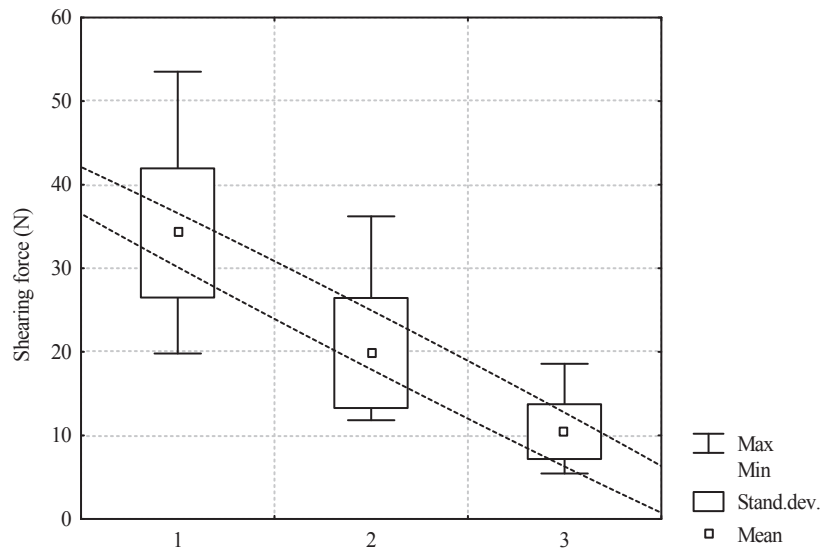

Figure 20. Cutting energy for different harvest terms: 1 - term I, 2 - term II, 3 - term III

The kernels are yellow-gold in the late milk ripeness. The period of corncobs suitability for consumption is relatively short - cobs get over-ripe rapidly, especially at high temperature. The process of over-ripening connected with transformation of sugars into starch, is delayed in with leaves and in cool conditions (Szymanek et al. 2005).

In order to estimate the effect of storage conditions on the possibility of extending the period of corn suitability for processing, tests were performed, consisting in storing corncobs for 7 days under the following conditions:

- conventional storage - temperature approx. $20^{\circ} \mathrm{C}$ (barn, umbrella roof),

- refrigerated storage - cold storage room, temperature approx. $2^{0} \mathrm{C}$,

- soaking in water - temperature approx. $20^{\circ} \mathrm{C}$.

Immediately after harvest the mechanical properties of cobs at late milk ripeness phase were compared, as well as those of cobs after blanching. Corncob blanching was made in a 4-minute 


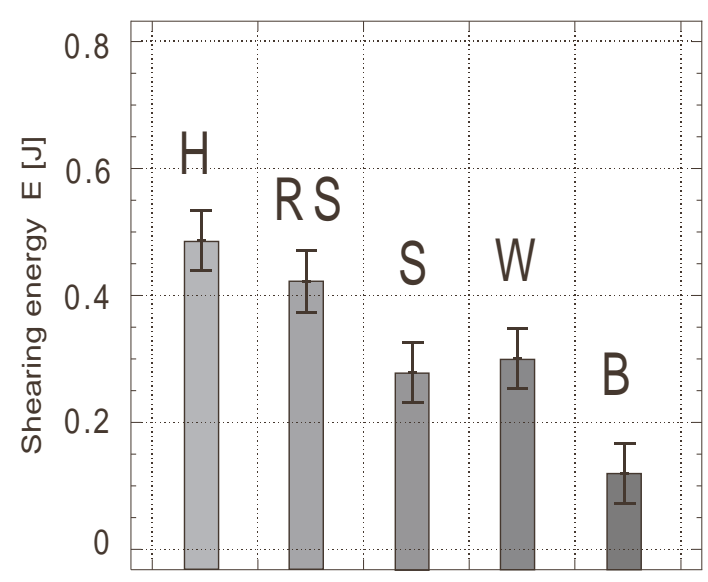

Figure 21. Energy of kernel cutting off from cob core. $\mathrm{H}$ - after harvest, $\mathrm{W}$ - wetted cobs, $\mathrm{S}$ - cobs from regular storage, RS - cobs from refrigerated storage and B - after blanching

water bath with a temperature of $80-90^{\circ} \mathrm{C}$. After the blanching, the cobs were cooled to a temperature of about $20^{\circ} \mathrm{C}$ and dried. As follows from Figure 21, kernels from cobs subjected to the process of blanching need lower energy expenditure for the kernel cutting process (0.27J), which provided an inducement for a broader study of the process of cutting of blanched kernels.

\section{Author details}

B. Dobrzański and A. Stępniewski

Institute of Agrophysics Polish Academy of Sciences, Lublin, Poland

\section{References}

[1] Abbott, J. A, \& Harker, R. (2003). Texture, http://usna.usda.gov/ hb66/021texture.pdf) , 1-19.

[2] Barbosa-Cánovas, G. V, Juliano, P, \& Peleg, M. (2004). Rev.2006), Engineering Properties of Foods, in Food Engineering. In: Barbosa-Cánovas, G.V., (ed.) Encyclopedia of Life Support Systems (EOLSS), Eolss Publishers, Oxford,UK, (http:// www.eolss.net) 
[3] Bilanski, W, Szot, B, Kushwaha, R. L, \& Stepniewski, A. Comparition of strength features of rape siliques and seeds for varieties cultivated in various countries. International Agrophysics, (1994). , 1994(8), 177-184.

[4] Chen, P, \& Sun, Z. (1991). A review of non-destructive methods for quality evaluation and sorting of agricultural products. Journal of Agricultural Engineering Research, 49: 85--98.

[5] Chen, P. (1996). Quality evaluation technology of agricultural products. Proc. of ICAME'96, Seul, Korea, Vol. I, , 171-190.

[6] Davison, E, Meiering, A. G, \& Middendorf, F. J. (1979). A theoretical stress model of rapeseed. Canadian Agricultural Engineering, , 21(1)

[7] Dobrzański, jr. B., (1993). Geometrical dependences of seed shell of compressed pea. Int. Agrophysics, , 7(4), 259-264.

[8] Dobrzański, jr. B., (1996). The influence of moisture content on the shape deformation of soybean. AgEng'96, Madrid, Paper 96F- , 082, 1-7.

[9] Dobrzański, jr. B., (2000). The analysis of seed cracking at grain legume processing. CD-ROM of AgEng'2000 papers, Paper: 00-PH- , 050, 1-10.

[10] Dobrzański, jr. B., (2002). The cracking mechanisms of grain legume. rozdział- str.: in "Physical Methods in Agriculture", J. Blahovec, M. Kutilek (eds): Kluwer Academic Publishers., 167-194.

[11] Dobrzański, jr., B. (2011). Shrinkage and swelling phenomena in agricultural products. in Encyclopedia of Agrophysics. J. Gliński, J. Horabik, J. Lipiec (eds.) Springer, 730-733.

[12] Dobrzański, jr. B., Szot B., (1997). Strength of pea seed cover. Int. Agrophysics, , 11(4), 301-306.

[13] Dobrzański, jr. B., (1998). Mechanizmy powstawania uszkodzeń nasion roślin strączkowych. Acta Agrophysicca, in polish), 13, 1-96.

[14] Dobrzański, jr. B., Banak E., Grundas S., Sosnowski S., Pecen J., (2003). Metoda rentgenograficzna $\mathrm{w}$ identyfikacji uszkodzeń wewnętrznych nasion fasoli szparagowej. Acta Agrophysica, 95, in polish), 2

[15] Dobrzański, jr. B., Mazurek W., Rybczyński R., Geodecki M., Baranowski P., Walczak R., (2003). A new method of the seed viability estimation. New Methods, Means and Technologies for Application of Agricultural Products. Agricultural Engineering LUA, 1(06), 105-110.

[16] Dobrzanski, B, \& Stepniewski, A. (1991). Modulus of elasticity of rapeseed shell. Proceedings of th. International Rapeseed Congress, Saskatoon, Canada, 1256-1260, 8. 
[17] Dobrzanski, B, Szot, B, \& Stepniewski, A. Stress in the shell during large deformation of compressed rapeseed. Proceedings of International Conference on Agricultural Engineering, Beijing, China, (1992).

[18] Dorell, D. G, \& Adams, M. W. (1969). Effect of some seed characteristic on mechanically included seedcoat damage in navy beans. Agron. J., , 61(5), 672-673.

[19] Fornal, J, Sadowska, J, \& Ostaszyk, A. (1995). Industrial drying of rapeseeds vs. oil extractivity. Proceedings of 9th. International Rapeseed Congress, Cambridge, England IV, , 1406-1408.

[20] Fornal, J, Sadowska, J, Jaroch, R, Kaczynska, B, \& Winnicki, T. (1994). Effect of drying of rapeseeds on their mechanical properties and technological usability. International Agrophysics, , 8

[21] Haman, J, Dobrzanski, B, Szot, B, \& Stepniewski, A. (1994). Strength of shell in compression test of rapeseed. International Agrophysics, , 8, 245-250.

[22] Ling ChY., Whitney L., (1975). Analysis of spherical shell acted on by two concentric forces in opposite directions. Theory, determination and control of physical properties of food materials., D. Reidel Publ. Co., , 189-196.

[23] Liu, M, Haghighi, K, \& Stroshine, R. L. (1989). Viscoelastic characterization of the soybean seedcoat. Trans. ASAE , 32(3), 946-952.

[24] Mac Donald, jr. M.B., (1985). Physical seed quality of soybean. Seed Sci. Technol. , 13, 601-628.

[25] Mensah, J. K, Nelson, G. L, Herum, F. L, \& Richard, T. G. (1984). Mechanical properties related to soybean seedcoat cracking during drying. Trans. ASAE, , 27(2), 550-560.

[26] Misra, R, Young, J. H, \& Haman, D. D. (1981). Finite element procedures for estimating shrinkage stresses during soybean drying. Trans. ASAE , 24(3), 751-753.

[27] Mohsenin, N. N. Physical properties of plant and animal materials. Gordon and Breach Science Publishers, New York, London, Paris (1978).

[28] Otten, L, Brown, R, \& Reid, W. S. (1984). Drying of white beans-effect of temperature and relative humidity on seed coat damage. Canad. Agric. Eng., , 26, 101-104.

[29] Overhults, D. G, White, H. E, Hamilton, H. E, \& Ross, I. J. (1973). Drying soybeans with heated air. Trans. ASAE , 16(1), 112-113.

[30] Palusen, M. R, Nave, W. R, \& Gray, L. E. (1981). Soybean seed quality as affected by impact damage. Trans. ASAE , 24(6), 1577-1582.

[31] Stepniewski, A, \& Dobrzanski, B. Higher temperature of rapeseeds as a factor affecting their mechanical resistance. Proceedings of 8-th. International Rapeseed Congress, Saskatoon, Kanada, (1991). , 1991, 1246-1250. 
[32] Stepniewski, A, \& Szot, B. Postharvest operations and quality of rapeseed. Proceedings of 9th. International Rapeseed Congress, Cambridge, England (1995). I, , 232-234.

[33] Stepniewski, A, \& Szot, B. Quality of rapeseed in postharvest handling. Preprints of workshop on Control Applications in Post-Harvest and Processing Technology, CAPPT'95, Ostend, Belgium (1995).

[34] Stepniewski, A, Szot, B, Fornal, J, \& Sadowska, J. Drying conditions and mechanical properties of rapeseed. J. Food Physics, (1994). , 1994, 86-89.

[35] Szot, B, \& Stepniewski, A. Studies on mechanical properties of rape in the aspect of its quantity and quality losses. Zemedelska Technika, (1995). , 1995(41), 133-136.

[36] Szot, B, Stepniewski, A, Dobrzanski, B, \& Rybczynski, R. The influence of higher temperature of rapeseeds on their mechanical resistance. Proceedings of th. International Conference Physical Properties of Agricultural Materials and Their Influence on Technological Processes, Rostock, Germany, (1989). s. 815- 818, 4.

[37] White, G. M, Bridges, T. C, Loewer, O. J, \& Ross, I. J. (1980). Seed coat damage in thin-layer drying of soybeans. Trans. ASAE , 23(1), 224-227. 